\title{
Minimalism in Radiation Synthesis of Biomedical Functional Nanogels
}

Clelia Dispenza, ${ }^{* \dagger}, \dagger$ Maria Antonietta Sabatino, ${ }^{\dagger}$ Natascia Grimaldi, $^{\dagger}$ Donatella Bulone, ${ }^{\ddagger}$ Maria Luisa Bondì, ${ }^{\S}$ Maria Pia Casaletto, ${ }^{\S}$ Salvatrice Rigogliuso," Giorgia Adamo," and Giulio Ghersi"

${ }^{\dagger}$ Dipartimento di Ingegneria Chimica, Gestionale, Informatica, Meccanica, Università degli Studi di Palermo, Viale delle Scienze Ed. 6, 90128 Palermo, Italy

${ }^{\ddagger}$ CNR - Istituto di Biofisica (IBF) UOS Palermo, via Ugo La Malfa 153, 90146 Palermo, Italy

${ }^{\S}$ CNR - Istituto per lo Studio dei Materiali Nanostrutturati (ISMN) UOS Palermo, via Ugo La Malfa, 153, 90146 Palermo, Italy "Dipartimento di Scienze e Tecnologie Molecolari e Biomolecolari, Università degli Studi di Palermo, Viale delle Scienze, Ed. 16, 90128 Palermo, Italy

Supporting Information

ABSTRACT: A scalable, single-step, synthetic approach for the manufacture of biocompatible, functionalized micro- and nanogels is presented. In particular, poly $(N$-vinyl pyrrolidone)-grafted-(aminopropyl)methacrylamide microgels and nanogels were generated through e-beam irradiation of PVP aqueous solutions in the presence of a primary amino-group-carrying monomer. Particles with different hydrodynamic diameters and surface charge densities were obtained at the variance of the irradiation conditions. Chemical structure was investigated by different spectroscopic techniques. Fluorescent variants were generated through fluorescein isothiocyanate attachment to the primary amino groups grafted to PVP, to both quantify the available functional groups for bioconjugation and follow nanogels localization in cell cultures. Finally, a model protein, bovine serum albumin, was conjugated to the nanogels to demonstrate the attachment of biologically relevant molecules for targeting purposes in drug delivery. The described approach provides a novel strategy to fabricate biohybrid nanogels with a very promising potential in nanomedicine.

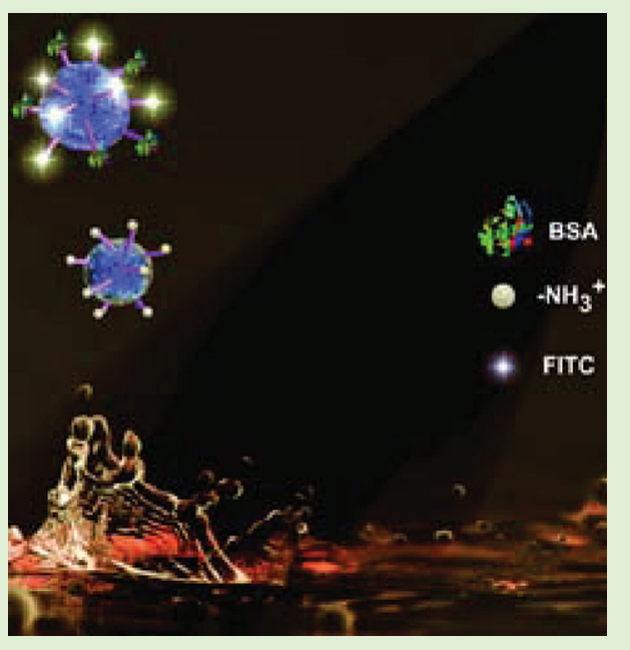

\section{INTRODUCTION}

Hydrogels have been often proposed as matrices for incorporation and controlled release of drugs, owing to their benign toxicological profile, soft and rubbery consistency, tailored chemical and physical properties. ${ }^{1-4}$ Further opportunities are offered by the control of size and shape of hydrogels down to the micro and the nanoscale. Differently from "gels", that is, macroscopic networks, their nanometric/micrometric analogues, that is, "nanogels" (NGs) or "microgels" (MGs), may "dissolve" in solvents, just as linear macromolecules do, yet preserving an almost fixed molecular conformation. They may swell in aqueous environments and respond to stimuli, such as temperature, $\mathrm{pH}$, redox potentials, and other modifications of the environmental conditions. ${ }^{5,6}$

Pharmaceutical NGs bearing reactive groups can represent a convenient alternative to the classical pharmacological treatment for several diseases, particularly in cancer chemotherapy, owing to a more specific and efficient particle surface functionalization, drug uptake, and release features. ${ }^{7}$ One of the main problems associated with the administration of pharmaceutical drugs, in fact, depends on the nonspecificity in recognition of the target sites and consequent homogeneous distribution of the drug in the body. ${ }^{8,9}$ Functionalized NGs, unlike other biomaterials, offer the unique advantages stemming from spacious aqueous interiors, high surface areas, and tunable, and thereby highly specific, chemical and physical characteristics. The high surface area enables us to bind the carrier with appropriate ligands, creating a system that can improve the localization of the drug near or at the target site. Localization in specific sites can be realized either through "passive targeting", when it is entrusted only to the best match between morphological, chemical, and physical properties of the carrier and the anatomical and physiological characteristics of the target site, or through "active targeting", that is realized by binding to the carrier rather appropriate moieties or entire molecules able to interact with membrane receptors on cells of the target site. The availability of inexpensive and robust preparation methodologies of such nanocarriers is at the basis of the development of effective NG-based theragnostic devices, and it has been the main limitation to their development. ${ }^{10}$

Received: February 27, 2012

Revised: $\quad$ May 2, 2012

Published: May 10, 2012 
Scheme $1^{a}$

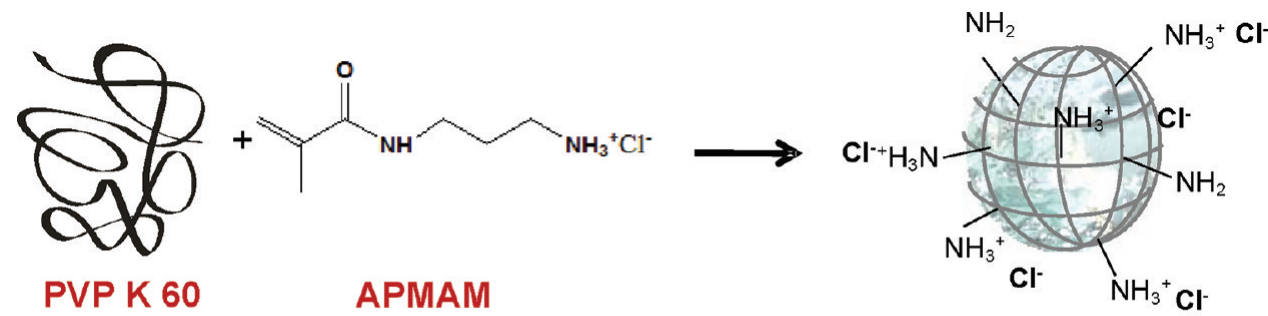

(a)

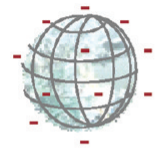

$P^{*}$

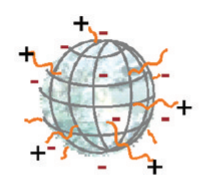

$P^{*}-g-A N G$

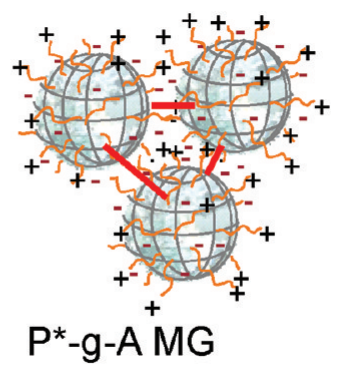

(b)

a (a) One-step synthesis of amino-functionalized PVP nanogel via e-beam irradiation. Both PVP and APMAM are present in the irradiated aqueous solution. PVP cross-linking and APMAM monomer grafting occur simultaneously. (b) Schematics of a typical base PVP nanogels (P*): the polymer network and negative surface charge density are evidenced; amino-grafted PVP ( $\mathrm{P}^{*}$-g-A) nanogels (NG) and microgels (MG). The curly orange lines represent grafted APMAM carrying a primary amino group. Bold red lines represent APMAM monomers or oligomers bridging cross-linked PVP coils.

Our radiation-engineered NGs are conceived to be produced with a process that ensures, simultaneously, polymer crosslinking, particles size control, chemical functionalization, and sterilization of the produced materials. They are characterized by a remarkable chemical and colloidal stability ${ }^{11}$ and amenability of subsequent decoration with a variety of specific ligands, thus combining the absence of toxicity with the specificity of effects only on selected targets. The structural integrity of the designed NGs will avoid interference between the incorporated therapeutics and polymeric carrier degradation products. It has already been established that NGs can be produced under high-dose pulse irradiation of dilute aqueous solutions of water-soluble polymers. ${ }^{12}$ Under these conditions polymer macroradicals form and recombine mainly within the same polymer coil, that is, intramolecularly. As a result, internally cross-linked single macromolecules are formed. Analogies and differences in cross-linking reaction mechanisms and kinetics underlying NGs formation have been discussed, ${ }^{13,14}$ but no adequate efforts have been paid so far in developing formulations based on these findings to generate multifunctional particles with the required properties for application as nanocarriers. With the present work, we have assessed the possibility of generating biocompatible, functionalized MGs and NGs using existing industrial-type electron accelerators and setups, that is, with a robust, economically viable and thereby industrially implementable process. Poly $(N$ vinyl-pyrrolidone) has been chosen as the base material for its known propensity to cross-link under irradiation in water and because it is widely used in pharmaceutical and cosmetic formulations. Water-soluble functional monomers can be added to the polymer aqueous solution before irradiation. Here we are presenting the results obtained with (3- $\mathrm{N}$-aminopropyl) methacrylamide hydrochloride, at different concentrations (see Scheme 1a), chosen to insert primary amino groups amenable to subsequent conjugation with either fluorescent or biological source molecules, for localization and active targeting purposes, respectively. The influence of process parameters on the control of particle size and the feasibility of derivatization reactions with fluorescein isothiocyanate (FITC) and BSA, as a model protein, were investigated.

\section{MATERIALS}

Chemicals. PVP K60 (Aldrich, $\left.M_{\mathrm{n}}=1.60 \times 10^{5} \mathrm{~g} / \mathrm{mol}\right),(3-\mathrm{N}$ aminopropyl) methacrylamide hydrochloride (APMAM, Polyscience), and FITC (Research Organics) were used without further purification. PVP's molecular weight, $M_{\mathrm{w}}=4.1 \times 10^{5} \mathrm{~g} / \mathrm{mol}$, and radius of gyration, $R_{\mathrm{g}}=27 \mathrm{~nm}$, were estimated from the usual Zimm plot analysis ${ }^{15}$ of static light scattering measurements, carried out at $25{ }^{\circ} \mathrm{C}$. Dialysis tubes of 12.000 and $100.000 \mathrm{Da}$ cutoff (Membrane Spectra/Por Biotech RC and CE, respectively, Spectrum Laboratories), $0.22 \mu \mathrm{m}$ pore size syringe filter (Millipore), and centrifuge filter device $10 \mathrm{kDa}$ cutoff (Centricon 10, Millipore) were used.

Cell Culture Reagents. The osteoblastic cell line MC3T3-E1 from a C57BL/6 mouse calvaria was grown in Dulbecco's modified Eagle's medium (DMEM) supplemented with 10 wt \% fetal calf serum (FCS; Euroclone, Celbio), 1 wt \% antibiotic, and 1 wt $\%$ glutamine (Euroclone, Celbio).

\section{METHODS}

Poly(N-vinyl pyrrolidone)-grafted-(aminopropyl) Methacrylamide Micro/Nanogels Synthesis. PVP aqueous solutions at $0.1 \mathrm{wt} \%$ (equivalent to $\sim 0.9 \times 10^{-2} \mathrm{~mol}$ of repetitive unit (RU) per liter, respectively) with APMAM (0.045, 0.09, 0.18, and $0.36 \mathrm{mM}$, corresponding 1:200, 1:100, 1:50, and 1:25 APMAM/PVP's RU molar ratios, respectively) and without APMAM (base PVP NGs) were prepared by overnight stirring, filtered with $0.22 \mu \mathrm{m}$ pore size syringe filters, carefully deoxygenated with gaseous nitrogen, and bottled in 
glass vials sealed with rubber septa and aluminum caps. Samples were individually saturated with $\mathrm{N}_{2} \mathrm{O}\left(\mathrm{N}_{2} \mathrm{O} \geq 99.99 \%\right)$ prior to irradiation to increase the concentration of hydroxyl radicals formed from water radiolysis during irradiation. ${ }^{16}$

Electron beam irradiation was performed using two $10 \mathrm{MeV}$ linear accelerators at the ICHTJ of Warsaw (Poland), LAE 13/ 9, and Electronika 10/10. For irradiation runs with LAE 13/9, parameters were set to have an average beam current of 0.40 $\mu \mathrm{A}$, pulse length of $10-12 \mu \mathrm{s}$, and pulse repetition rate of 75 $\mathrm{Hz}$. The dose per pulse was $\sim 1.8 \mathrm{~Gy}$, corresponding to $\sim 500$ $\mathrm{kGy} / \mathrm{h}$. Samples irradiated under these conditions were named after "LX-500", where $\mathrm{X}$ is a number indicating the total absorbed dose in kGy. The homogeneous dose distribution and the integrated absorbed dose by the sample were measured using cellulose triacetate film dosimeters on the basis of absorbance at $298 \mathrm{~nm}$. Sample vials were placed horizontally in front of the electron emission port in a suitable container filled with ice. Temperature during irradiation was measured to be maintained between 5 and $10{ }^{\circ} \mathrm{C}$.

Irradiation with Elektronika $10 / 10$ was carried out at an average beam current of $0.45 \mathrm{~mA}$, pulse length of $4.5 \mu \mathrm{s}$, and pulse repetition rate of $300 \mathrm{~Hz}$. In this case, dosimetry was performed using a graphite calorimeter; the measuring error was $\pm 4 \%$. Samples were horizontally placed in a box filled with ice and conveyed under the beam via a transporting belt at a speed of $0.3 \mathrm{~m} / \mathrm{min}$. An integrated dose of $40 \mathrm{kGy}$ was supplied with a single pass, whereas the higher dose investigated here (80 kGy) was obtained with a double-pass exposure. The total absorbed dose results from a combination of e-beam accelerator's setup parameters and speed of the conveyer's belt. From a measurement of the residence time of the vials under the beam $(\sim 10 \mathrm{~s})$ we can estimate an approximate absorbed dose per pulse of $\sim 13$ Gy. Samples irradiated under these conditions were named after "EX", were $\mathrm{X}$ is the total absorbed dose in kGy.

After irradiation, samples were dialyzed against distilled water for $48 \mathrm{~h}$. Dialysis was initially performed with two different membranes: $12 \mathrm{k}$ MWCO and 100k MWCO. Samples recovered from dialysis were subjected to light scattering measurements to compare the intensity of scattered light. Results are reported as Figure S1 of the SI. pH of the systems was measured before and after irradiation and after dialysis at the controlled temperature of $25 \pm 1{ }^{\circ} \mathrm{C}$ with CRISON GLP $22 \mathrm{pH}$ and ion meter. All formulations had a $\mathrm{pH}$ of 6.0 to 7.0 prior to irradiation, which turned out to be in the range 3.1 to 4.5 after irradiation and between 4.5 to 5.8 after dialysis.

Product Yield Determination. The yield of the micro/ nanogels production process was determined gravimetrically as the weight ratio of recovered product after irradiation, dialysis (MWCO 12k), and freeze-drying and the corresponding initial weight of the polymer and the monomer loaded to the vial. Measurements were always carried out in triplicate from independent irradiation runs.

By dialysis, we expect to remove eventual low-molecularweight fragments eventually deriving from PVP degradation and APMAM monomer not grafted to the polymer. Homopolymerization of APMAM should be minimal under the selected irradiation conditions, owing to (i) the low concentration of monomer $\left(4.5 \times 10^{-5}\right.$ to $3.6 \times 10^{-4} \mathrm{M}$ vs 0.9 $\times 10^{-2} \mathrm{M}$ of PVP RU) and (ii) the low $\mathrm{pH}$ (always lower than APMAM's $\left.\mathrm{pK}_{\mathrm{a}}=8.5\right) .{ }^{17}$ Repulsive forces acting between charged monomers should effectively prevent them coming into proximity and react. This hypothesis is supported by light scattering and ${ }^{1} \mathrm{H}$ NMR data collected on irradiated APMAM solutions. (For details, see Figure S2 of the SI.)

Spectroscopic Characterizations. FTIR analysis was carried out with Perkin-Elmer-Spectrum 400 apparatus by dispersing the dry product in potassium bromide and compressing into pellets. Spectra were recorded at 30 scans per spectrum and $1 \mathrm{~cm}^{-1}$ resolution in the $4000-400 \mathrm{~cm}^{-1}$ range.

${ }^{13} \mathrm{C}\left\{{ }^{1} \mathrm{H}\right\}$ CP-MAS NMR analysis was performed on solid samples with a Bruker Avance II $400 \mathrm{MHz}(9.4 \mathrm{~T})$ spectrometer operating at $400.15 \mathrm{MHz}$ for the ${ }^{1} \mathrm{H}$ nucleus and $100.63 \mathrm{MHz}$ for the ${ }^{13} \mathrm{C}$ nucleus with a MAS rate of 13 $\mathrm{kHz}, 1024$ scans, a contact time of $1.5 \mathrm{~ms}$ and a repetition delay of $2 \mathrm{~s}$. The optimization of the Hartmann-Hahn condition was obtained using an adamantane standard. All samples were placed in $4 \mathrm{~mm}$ zirconia rotors with KEL-F caps. Silica powder was mixed with the samples to obtain a more compact filling of the rotor's volume.

Surface chemical composition of the samples was investigated by XPS in an ultrahigh vacuum (UHV) chamber with a base pressure in the range of $10^{-8}$ Torr during data collection. Photoemission spectra were collected by a VG Microtech ESCA 3000 Multilab spectrometer, equipped with a standard Al $\mathrm{K}_{\alpha}$ excitation source $(h \nu=1486.6 \mathrm{eV})$ and a nine-channeltrons detection system. The hemispherical analyzer operated in the CAE mode, at a constant pass energy of $20 \mathrm{eV}$. The binding energy (BE) scale was calibrated by measuring $\mathrm{C} 1 \mathrm{~s}$ peak $(\mathrm{BE}=$ $285.1 \mathrm{eV}$ ) from the surface contamination, and the accuracy of the measure was $\pm 0.1 \mathrm{eV}$. Photoemission data were collected and processed by using the VGX900 software. Data analysis was performed by a nonlinear least-squares curve-fitting program using a properly weighted sum of Lorentzian and Gaussian component curves, after background subtraction according to Shirley and Sherwood. ${ }^{18}$ Surface relative atomic concentrations were calculated by a standard quantification routine, including Wagner's energy dependence of attenuation length ${ }^{19}$ and a standard set of VG Escalab sensitivity factors. The uncertainty on the atomic concentration is of the order of $10 \%$.

Light Scattering Measurements. The hydrodynamic radius $\left(R_{\mathrm{h}}\right)$ of particles dispersion was measured by dynamic light scattering (DLS) using a Brookhaven Instruments BI200SM goniometer. Samples, placed in the quartz cell after dilution $(0.025 \mathrm{mg} / \mathrm{mL})$ with bidistilled water, were put in the thermostatted cell compartment of the instrument at $20 \pm$ $0.1{ }^{\circ} \mathrm{C}$. Intensity autocorrelation function at the scattering angle of $90^{\circ}$ and time autocorrelation function were measured by using a Brookhaven BI-9000 correlator and a $50 \mathrm{~mW} \mathrm{He}-\mathrm{Ne}$ laser (MellesGriot) tuned at $\lambda=632.8 \mathrm{~nm}$. The correlator was operated in the multi- $\tau$ mode; the experimental duration was set to have at least 2000 counting on the last channel of the correlation function. All irradiated samples were analyzed as produced, without filtration, to minimize artifacts. Particular care was paid in treating the samples in a clean environment to reduce contamination. No influence of $\mathrm{pH}$ on the decay curves when varying from 4 to 6 was observed by the different systems (data here not reported for brevity). In consideration of the fact that the samples showed a monomodal distribution, DLS data were analyzed by the method of cumulants. According to this method, the logarithm of the field-correlation function is expressed in terms of a polynomial in the delay time $\tau$, with the first and second cumulant providing information on the mean value and standard deviation of the distribution of nanoparticles 
hydrodynamic size. ${ }^{15,20}$ Measurements were carried out on a minimum two samples from three independent runs. For each formulation and irradiation condition analyzed, very similar results were obtained in terms of particle size distribution. The weight-average molecular weight $\left(M_{\mathrm{w}}\right)$ and radius of gyration of PVP in aqueous solution at $25^{\circ} \mathrm{C}$ were estimated from Zimm plot of static light scattering measurements at different scattering angles and polymer concentration. The refractive index increment $(\mathrm{d} n / \mathrm{d} c)$ of PVP in aqueous solution was measured by using a Brookhaven Instruments differential refractometer at $\lambda=620 \mathrm{~nm}$. We obtained the value of $0.18 \pm$ $0.004 \mathrm{~mL} / \mathrm{g}$ in good agreement with a literature value. ${ }^{21}$ When attempting to draw a Zimm plot with scattered light data collected on NGs samples, a strong downward curvature in the scattering angle dependence was observed, probably due to polydispersity and interparticles interactions. This occurrence prevented a reliable determination of extrapolated light scattered values at zero scattering angle. However, a rough estimate of the molecular weight in the case of particular interest was obtained by averaging the values obtained through Debye's plots for each different scattering angle. To determine the relative gyration radius, each data set at different concentration was analyzed in terms of the Guinier's expression: $1 / P(q)=1+\left(q R_{\mathrm{g}}\right)^{2} / 3+\Theta(q)$, where $P(q)=$ $I(q) / I(q=0)$ and $q=4 \pi n / \sin (\theta / 2)$. Taking $q^{2}$ as an independent variable, a polynomial fit of $1 / P(q)$ was applied by considering only the points at low $q$ value, and $R_{\mathrm{g}}$ was derived by the first-order coefficient.

$\zeta$-Potential Measurements. The surface charge of the NGs in water was measured according to the principles of laser Doppler velocitometry and phase analysis light scattering (M3PALS technique) and expressed as $\zeta$ potential. It was measured on diluted dispersions $(0.025 \mathrm{mg} / \mathrm{mL})$ at $25{ }^{\circ} \mathrm{C}$ using a ZetaSizerNano ZS (Malvern Instruments, Malvern, U.K.) equipped with a $\mathrm{He}-\mathrm{Ne}$ laser at a power of $4.0 \mathrm{~mW}$. As for particle size distribution, also $\zeta$ potential measurements were carried out on minimum two samples from three independent runs. Error bars reported in the $\zeta$ potential plot refer to the width of the $\zeta$ potential distribution for each sample, which was fairly high for some formulations.

Preparation and Characterization of Fluorescent Micro/Nanogels Variants. A given volume $(0.6 \mathrm{~mL})$ of MG or NG dispersion was mixed with $0.05 \mathrm{M}$ Borax ( $\mathrm{pH}$ 9.3) and $0.4 \mathrm{M} \mathrm{NaCl}$ aqueous solution $(1.4 \mathrm{~mL})$ and with $14 \mu \mathrm{g} / \mathrm{mL}$ methanol/water solution $(0.1 \mathrm{vol} \% \mathrm{MeOH})$ of FITC $(2 \mathrm{~mL})$. The solution was kept at $37^{\circ} \mathrm{C}$ while stirring for $2 \mathrm{~h}$. The FITC conjugated MGs or NGs were then thoroughly dialyzed against $\mathrm{pH}$ 7.4 PBS, using a dialysis tubing (MWCO 12-14 kDa), refreshing the solutions twice a day. Different dialysis times were investigated to ascertain that unbound FITC was effectively removed. In general, after $72 \mathrm{~h}$ of dialysis, unreacted FITC was removed. With the aim of quantitatively estimating the concentration of reacted amino groups for selected conjugated systems, FITC detachment from $\mathrm{P}^{*}$-g-A NGs was performed by changing the $\mathrm{pH}$ of the conjugated NGs' dispersions from 7.4 to 12 , adding a small quantity of $\mathrm{NaOH}$ (1M). Dispersions were kept stirred at $\mathrm{pH} 12$ and room temperature for $2 \mathrm{~h}$. Afterward, the $\mathrm{pH}$ was brought back to 7.4, and the detached FITC was separated from the NGs using a centrifuge filter device (cut off $10 \mathrm{kDa}$ ) at a speed of $4000 \mathrm{~g}$. The solution of FITC collected under the filter and the NGs retained by the filter and dispersed in a note volume of $\mathrm{pH} 7.4$ PBS was analyzed. In particular, UV-visible absorption and fluorescence spectroscopy were carried out on (i) the conjugated amino-functionalized MG or NG dispersions ( $\mathrm{P}^{*}$ $\left.\mathrm{g}-\mathrm{A}^{\mathrm{FITC}}\right)$; (ii) the nonconjugated analogues as negative controls ( $\mathrm{P}^{*}$-g-A); (iii) free probe solutions as positive controls or solutions separated from NGs after detachment (FITC sol); and (iv) base PVP systems subjected to the same protocols applied for reacting FITC with $\mathrm{P}^{*}$-g-A variants $\left(\mathrm{P}^{* \text { FITC }}\right)$.

UV-visible absorption measurements were carried out with a Shimadzu 2401-PC spectrofluorimeter (scan speed $40 \mathrm{~nm} / \mathrm{min}$, integration time $2 \mathrm{~s}$, bandwidth $1 \mathrm{~nm}$ ) at room temperature. Fluorescence spectra were acquired with a JASCO FP-6500 spectrofluorimeter, equipped with a Xenon lamp (150 W). Emission spectra, at the required excitation wavelength, were obtained with emission and excitation bandwidth of 1 and 3 $\mathrm{nm}$, respectively. Samples were excited at the maximum absorption wavelength for FITC $\left(\lambda_{\text {ex }} \cong 490 \mathrm{~nm}\right)$. Emission spectra were normalized with respect to the absorption at the excitation wavelength.

Preparation of BSA Conjugated Variants. P*-g-A(100) NGs dispersion $(1.2 \mathrm{~mL})$ was incubated with an equal volume of Borax solution (0.05 M - pH 9.3) with $\mathrm{NaCl}(0.4 \mathrm{M})$ for 10 min at room temperature. An aqueous solution $(600 \mu \mathrm{L})$ containing $1 \mathrm{mg}$ of ultrapure bovine serum albumin (BSA; Sigma-Aldrich) was added. The control system was also prepared as described above, but BSA was not added. The mixtures were then incubated at $37{ }^{\circ} \mathrm{C}$ for $2 \mathrm{~h}$, while shaking. Afterward, both BSA conjugated systems $\left(\mathrm{P}^{*}-\mathrm{g}-\mathrm{A}^{\mathrm{BSA}}\right.$ ) and relative control were thoroughly dialyzed against PBS using 100 $\mathrm{kDa}$ membranes, to allow the release of nonconjugated BSA. To confirm the occurrence of conjugation, samples were tested by a DU 730 Life Science spectrophotometer (Beckman Coulter) in a range between 200 and $500 \mathrm{~nm}$. To quantify the amount of conjugated BSA to the nanoparticles, we built up a calibration curve using solutions with increasing concentrations of the protein. Nanogel-BSA conjugates were stored up to 4 weeks at $4{ }^{\circ} \mathrm{C}$ and subjected to UV-vis analysis after double washings with PBS, showing no appreciable changes in the spectra.

Caspase 3 Enzymatic Assay on Synthetic Fluorescent Substrate. MC3T3-E1 cells were seeded at high density on a six-well plate for $24 \mathrm{~h}$ in complete medium; then, they were grown for another $24 \mathrm{~h}$ after incubation with $\mathrm{P}^{*}$-g-A (100) NGs. Cells were enzymatically detached from culture plate using a solution of Trypsin-EDTA 1X (Sigma) and centrifuged at $1000 \mathrm{rpm}$ for $5 \mathrm{~min}$. Pelleted cells were then resumed in 70 $\mu \mathrm{L}$ of Triton X 100 (1\%) in PBS and incubated $10 \mathrm{~min}$ at room temperature; the extract cell proteins suspension was centrifuged at $10.000 \mathrm{rpm}$ for $10 \mathrm{~min}$; then. the pellet containing the cellular bodies and nonextracted portion was removed, while the amount of extracted proteins present in the supernatant was quantified by Bradford microassay method (Bio-Rad, Segrate, Milan, Italy) employing BSA (SigmaAldrich) as standard.

MC3T3-E1 cells extracts $(20 \mu \mathrm{g})$, obtained as previously described, were used to detect the presence of activated caspases 3/7/8. In the assay, both untreated MC3T3-E1 cells (negative control) and $10 \mu \mathrm{M}$ Doxorubicin (DXR, known as apoptotic inducer)-treated cells for $4 \mathrm{~h}$ (positive control) were also used. The Ac-Asp-Glu-Val-Asp-MCA peptide (Pepta Nova, Peptide Institute), a specific substrate for activated caspase 3/ $7 / 8$ (typical apoptosis marker) ${ }^{22,23}$ was used. It ties a fluorophore in its cutting site, which emits fluorescence when activated by enzymes. In these experiments, the previously 
described extracts were added to $75 \mu \mathrm{L}(8 \mu \mathrm{M})$ of the substrate in a 96-well plate. By spectrofluorimetric reading, it was possible to quantify the degree of caspase activation. The spectrofluorimetric analysis was performed using Spectra Max Gemini EM-500 (Molecular Devices) and elaborated by Soft Max Pro 5.2 software.

MC3T3-E1 Cell Viability by MTT Assay. The MC3T3-E1 cells were seeded in a 96-well plate at density of $1 \times 10^{4}$ cell/ well. After $24 \mathrm{~h}$ of growth in the presence of the previously described dispersion, cells were washed with PBS and then incubated with $200 \mu \mathrm{L} /$ well of complete medium containing $0.25 \mathrm{mg} / \mathrm{mL}$ of MTT solution for $2 \mathrm{~h}$ at $37 \mathrm{C}$. After solubilization of the resulting formazan product with $100 \mu \mathrm{L} /$ well of DMSO solution, the absorbance of intense purple staining at $490 \mathrm{~nm}$ wavelength was read on a DU-730 Life Science spectrophotometer (Beckman Coulter). MC3T3-E1 cells treated with DXR ( $5 \mu \mathrm{M})$ for $24 \mathrm{~h}$ were used as positive control. Each experiment was repeated eight times.

Cellular Internalization Studies: Confocal Analysis. The MC3T3-E1 was grown at a density of $5 \times 10^{3}$ cells/well in 12 -well plates containing sterile coverslips in complete DMEM for $24 \mathrm{~h}$. Next, the cells were incubated with a given amount of $\mathrm{P}^{*}$-g-A $(100)^{\mathrm{FITC}} \mathrm{NGs}$ dispersion $(20 \mu \mathrm{g} /$ well $)$, which emits green fluorescence by FITC conjugation.

After different incubation times, respectively, $30 \mathrm{~min}, 1,3,6$, 8,24 , and $48 \mathrm{~h}$, the cells were washed twice with PBS to remove nanoparticles that were not taken up by the cells or were only loosely bound to the cellular membrane, fixed with $3.7 \mathrm{wt} \%$ formaldehyde for $15 \mathrm{~min}$, and washed again twice with PBS. Afterward, cells were stained with ethidium bromide (EtBr 1:1000, an intercalating agent commonly used as a fluorescent red tag to label the cell nucleus) for $1 \mathrm{~min}$ at room temperature. The nanoparticle position was monitored by confocal microscopy analysis (Olympus $1 \times 70$ with MellesGriot laser system); a $1 \mu \mathrm{m}$ thickness optical section was taken, and about 15 sections were taken for each sample.

Spectrofluorimetric Analysis of Fluorescent Nanoparticles Uptaken by Cells. The MC3T3-E1 cells were seeded in a 96-well tissue plate at a density of $7 \times 10^{3}$ cells/ well, grown for $24 \mathrm{~h}$ in DMEM complete medium, and incubated in the presence of $\mathrm{P}^{*}$-g-A $(100)^{\text {FITC }}(4 \mu \mathrm{g} /$ well $)$ NGs for different times $(0,30 \mathrm{~min}, 1,3,6,24$, and $48 \mathrm{~h})$. For each time, three wells were subjected to spectrofluorimetric readings (excitation at $485 \mathrm{~nm}$ and emission at $538 \mathrm{~nm}$ ) to measure the total fluorescence on the well; then, the cells were separated from the medium and washed twice with PBS. Fluorescence from the nanoparticles present inside the cells, in the separated medium, and in the washings was singularly evaluated using Spectra Max Gemini EM-500 (Molecular Devices, using the Soft Max Pro 5.2 software).

\section{RESULTS AND DISCUSSION}

Synthesis and Dimensional Characterization of Micro and Nanogels. It has been already demonstrated that electron beam irradiation applied to semidilute PVP aqueous solutions, at relatively high doses per pulse, can fix a specific polymer conformation in water through only a few intramolecular covalent bonds, thus leading to the formation of micro and nanogels. In $\mathrm{N}_{2} \mathrm{O}$-saturated aqueous solutions of PVP, carboncentered PVP radicals are promptly formed by the rapid $\mathrm{H}$ abstraction reaction by $\cdot \mathrm{OH}$ and $\cdot \mathrm{H}$, which are the predominant radical species formed in such solutions, with radical yields of approximately 0.58 and $0.06 \mu \mathrm{mol} \mathrm{J}{ }^{-1} .{ }^{16}$ The decay of PVP
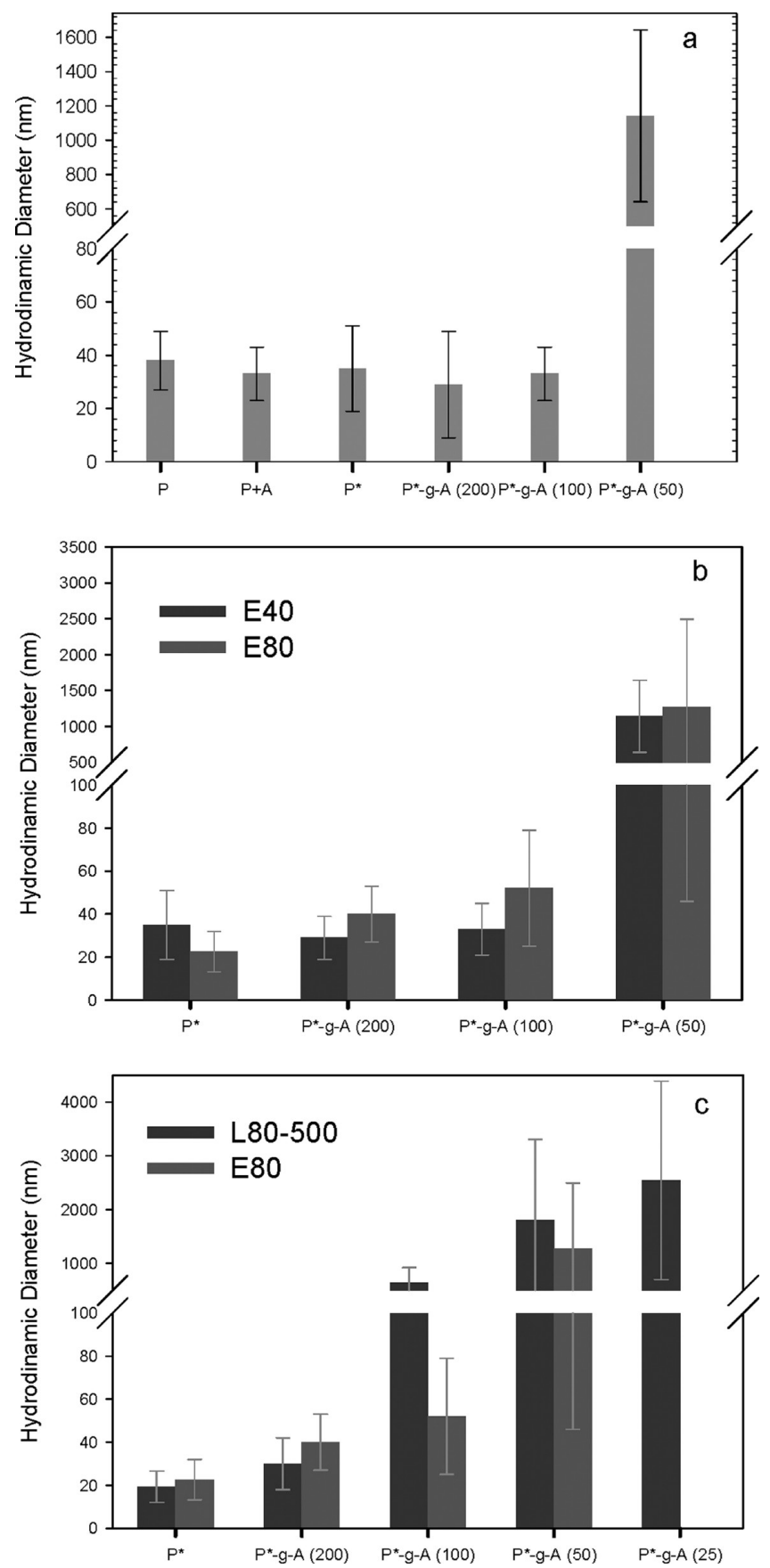

Figure 1. MGs and NGs hydrodynamic diameters from DLS analysis at $20{ }^{\circ} \mathrm{C}$ in water. (a) Systems irradiated with low dose-rate and the lower dose (E40). Nonirradiated aqueous PVP both in the presence and in the absence of APMAM is reported for comparison. (b) Influence of irradiation dose for the different formulations. (c) Influence of dose-rate for the different formulations. Error bars represent the variance of the particle size distribution.

radicals after a single pulse imparted to the linear polymer is generally faster than the time between two successive pulses. When the average number of radicals generated on each polymer chain upon a pulse significantly exceeds one, polymer radicals prevalently recombine intramolecularly. NGs can thus be obtained in correspondence of relatively low irradiation doses $(5-10 \mathrm{kGy})$. $^{1,12,14,24}$

If sterility has to be granted, as it would be desirable for biomedical application of such NGs, then higher irradiation 


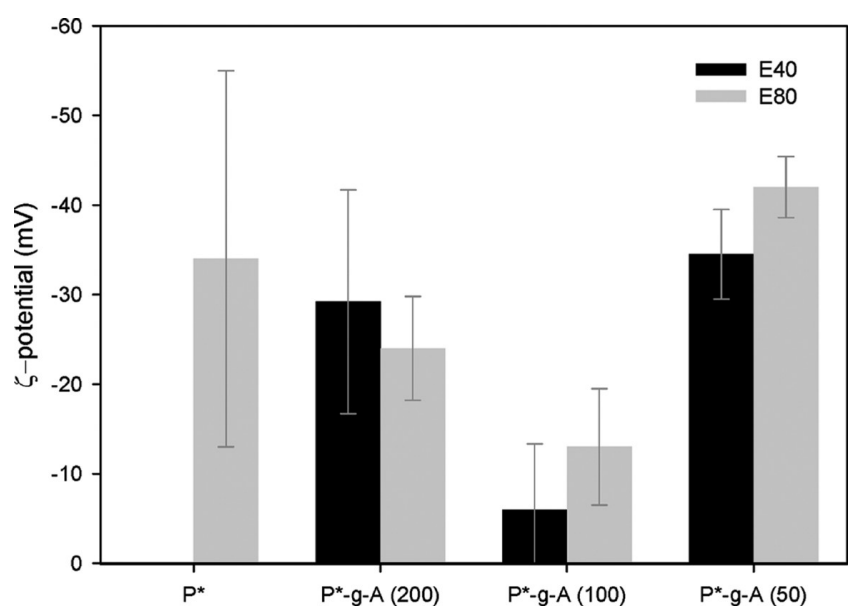

Figure 2. Surface charge density for the micro and nanogels produced at the different PVP RU/APMAM molar ratios and at the two doses of 40 and $80 \mathrm{kGy}$. Error bars represent the width of the surface charge distribution.

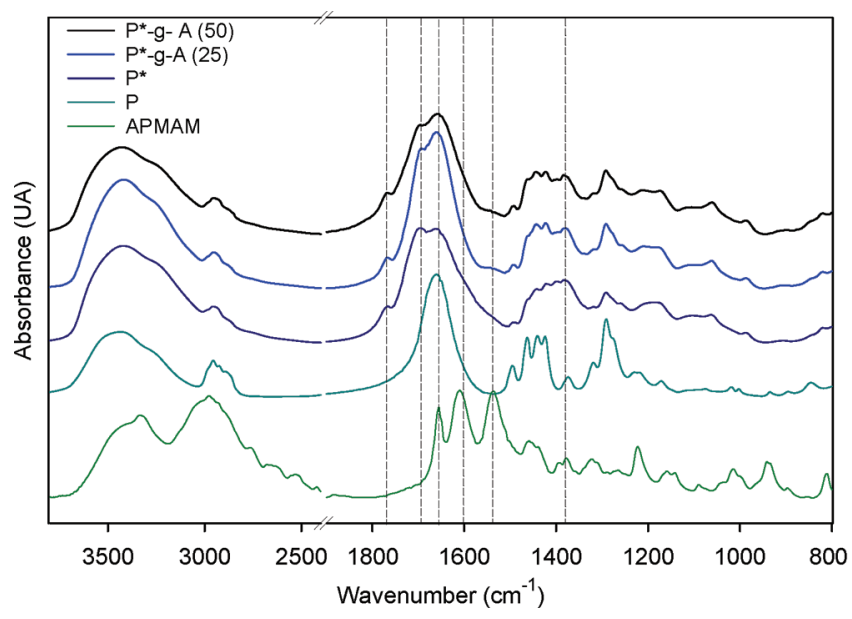

Figure 3. Comparison of FT-IR spectra of the nonirradiated PVP, base PVP nanogels $\left(\mathrm{P}^{*}\right)$ and amino-functionalized variants irradiated with LAE $13 / 9$ at $80 \mathrm{kGy}$. All spectra, with the exception of that of APMAM, were normalized with respect to the peak at $2956 \mathrm{~cm}^{-1}$. Vertical dashed lines correspond to the following peaks: 1661, 1695, $1765,1600,1537$, and $1388 \mathrm{~cm}^{-1}$.

doses would be required (20-40 kGy) and, for a more conservative approach, materials should also be tested at twice the anticipated maximum dose. The possibility of generating asborne sterile functional micro- or nanoparticles was investigated here by irradiating PVP/APMAM dilute aqueous solutions at total absorbed doses of 40 and $80 \mathrm{kGy}$, with the purpose of inducing simultaneously polymer cross-linking, monomer grafting to the cross-linked particles, and sterility, in a single step.

Two different irradiation conditions, that is, electron beam pulse frequency and dose per pulse, were selected: $300 \mathrm{~Hz}$ with $13 \mathrm{~Gy} /$ pulse and $75 \mathrm{~Hz}$ with $1.8 \mathrm{~Gy} /$ pulse, corresponding to two significantly different dose rates. Under the first set of conditions, irradiation of a $0.1 \mathrm{wt} \%$ of PVP leads to an approximate average number of radical centers for macromolecule equal to four, whereas under the latter conditions it is one every two chains. Under none of these conditions do we expect polymer radicals' intramolecular recombination to be the predominant reaction. The macromolecular constructs formed will be the result of the competition of several possible reactions: recombination of polymeric macroradicals, both intra- and intermolecularly, recombination of macroradicals with other radicals, disproportionation, chain scission, radical transfer though $\mathrm{H}$-abstraction, and so on. ${ }^{25}$ As we are moving in an unexplored territory of possible simultaneous acts of macromolecular structure disruption and reconstruction, the final product will be the result of an evolutionary process that will affect more the loosely cross-linked portions of the material and less the more densely networked ones. ${ }^{26,27}$ Micro/ nanoparticle structure and functionality will be controlled by the irradiation conditions and the gradual modification of the materials that is being irradiated.

In the presence of the acrylic monomer, we would expect that reactions of polymeric macroradicals can also involve APMAM, leading to monomer grafting (Scheme 1a). To confirm whether the overall approach can be successful, there are two possibilities: one is to attempt an identification of the radicals formed during the process and a kinetic analysis of their decay, the other is to perform an accurate product analysis. None of these two approaches is fast or simple. It has been preferred to collect first information about the usefulness of these micro- and nanostructures, which can then provide motivation to a deeper fundamental understanding of the reaction mechanisms.

The yield of the process, in terms of recovered dry product after dialysis, which may include both cross-linked and uncross-linked PVP and PVP-g-APMAM, was investigated gravimetrically after extensive dialysis and freeze-drying. For all irradiation conditions and formulations, the yield was always above 98 wt \%.

In Figure 1a, the average hydrodynamic diameter and relative standard deviations from DLS measurements carried out on the linear PVP, coded as "P", and the corresponding NG dispersions $\left(\mathrm{P}^{*}\right)$, as obtained after e-beam irradiation at the integrated dose of $40 \mathrm{kGy}$ and the highest dose rate (E40), are shown. No appreciable differences in the two average hydrodynamic diameters are observed. From a more careful examination of the scattered light decay curves (see Figure S3 of the SI), some differences can be appreciated: $\mathrm{P}^{*}$ is characterized by a larger proportion of smaller objects and few larger objects. This suggests that irradiation is causing a slight contraction of the great majority of polymer coils and simultaneously the formation of few bigger particles. The addition of APMAM to PVP (at the molar ratio 1:50 with respect to PVP RU) is causing a slight reduction of $D_{\mathrm{h}}(\mathrm{P}-\mathrm{A}$ system) already prior to irradiation. PVP presents ionic and/or polar negative charges owing to keto-enolic tautomerism of pyrrolidone carbonyls. Accordingly, the surface charge distribution for nonirradiated PVP macromolecules in doubledistilled water shows a main broad peak centered around neutrality and a second peak at about $-20 \mathrm{mV}$. (See Figure S4 of the SI.) Therefore, shrinkage of PVP coils is likely due to the shielding effect exerted by the protonated amino groups of APMAM toward the anionic charges of PVP. The average hydrodynamic diameters $\left(D_{\mathrm{h}}\right)$ of the particles obtained upon irradiation, at the variance of APMAM content, are also reported in Figure 1a. Poly $(N$-vinylpyrrolidone)-grafted-(aminopropyl) methacrylamide MGs or NGs are coded $\mathrm{P}^{*}$-g-A $(X)$, where is $X=200,100,50$, or 25 depending on the APMAM/ PVP's RU molar ratio.

Increasing APMAM content from 1:200 to $1: 100, D_{\mathrm{h}}$ only slightly increases, always leading to NGs of comparable size to 

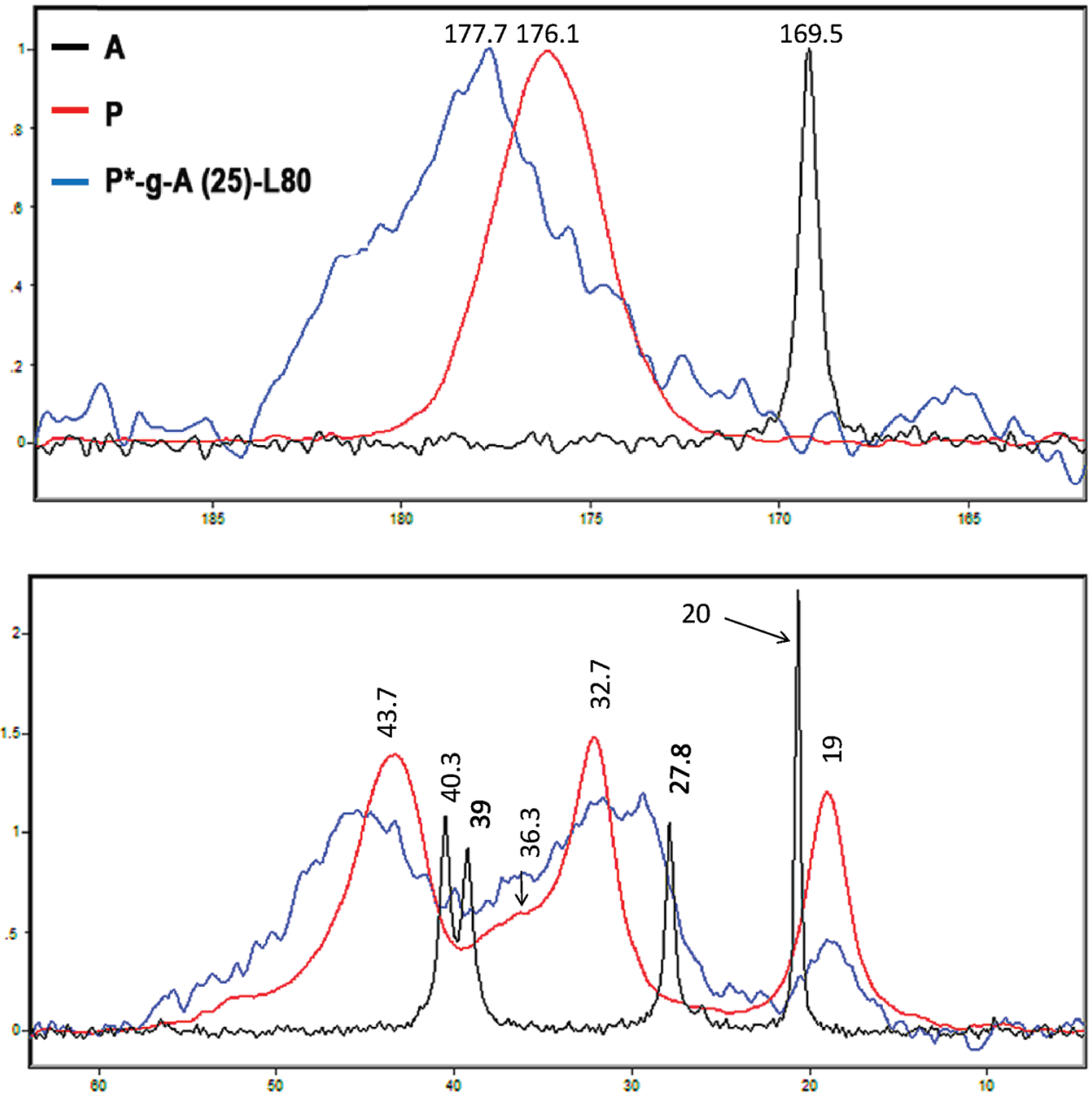

Figure 4. Overlaid ${ }^{13} \mathrm{C}\left\{{ }^{1} \mathrm{H}\right\}$ CP MAS NMR spectra of nonirradiated PVP (P), APMAM monomer (A), and amino-functionalized nanogels (P*-g-A (25)-L80) on an expanded scale. Spectra are normalized with respect to the carbonyl peak.

the $\mathrm{P}^{*}$ values. At 1:50 ratio, particle size suddenly increases to few micrometers. This sudden increase in $D_{\mathrm{h}}$ is likely to be associated with a step change in the main type of cross-linking reactions upon irradiation. When e-beam irradiation of the aqueous solutions of PVP with APMAM is carried out with both components present at low concentration, it induces grafting of APMAM to individual PVP nanoparticles, whereas at the increase in APMAM content interparticle bridging may concur (Scheme 1b). As a consequence, the stepwise increase in particles size is observed and microparticles are formed.

Figure $1 \mathrm{~b}$ compares the dimensions of particles obtained for the same pulse repetition rate and dose per pulse at two integrated doses: namely, 40 and $80 \mathrm{kGy}$. The effect of increasing the dose, from 40 to $80 \mathrm{kGy}$, for the base PVP systems is in the direction of reducing the NGs' particle size and narrowing the particle size distribution. In principle, this effect could be caused by either a high degree of intramolecular cross-linking, inducing of polymer coil shrinkage, or chain scission, that is, molecular degradation. To rule out any significant influence of radiation-induced molecular degradation at this fairly high irradiation dose, we carried out static light scattering measurements on this system at the variance of the scattering angle and sample concentration to determine the weight-average molecular weight. A marked downward curvature in the scattering angle dependence was observed in the case of $\mathrm{P}^{*}$, thus making unreliable the extrapolation procedure. The molecular weight and gyration radius were therefore estimated following the procedure described in the Materials and Methods sections. In the light of these measurements, one can observe that $M_{\mathrm{w}}$ varies from $4.20 \times$ $10^{5}( \pm 0.1)$ for the nonirradiated PVP to $9.62 \times 10^{5}( \pm 0.4)$ for $\mathrm{P}^{*}$ and the radius of gyration changes from 27 to $19 \mathrm{~nm}$. The observed increase in average molecular weight, accompanied by a reduction of $R_{\mathrm{g}}$ is the result of a densification of the material upon prolonged irradiation. Furthermore, irradiation at this dose induces a marked anionic character to the NGs, as it will be further discussed.

When it comes to comparing hydrodynamic diameters of systems obtained by irradiation of PVP in the presence of APMAM, higher doses generally lead to bigger particles that are still in the nanoscale range for the lower molar ratios (1:200 and 1:100), whereas they become micrometer-sized for the 1:50 ratio, with concomitant particle size distribution broadening. Under this last condition, prolonged irradiation acts as both an axe and a cement for the already formed microparticles, thus suggesting chain scission, macromolecular segments grafting, and cross-linking as concurrent phenomena. When the dose is imparted at the lower dose rate (L80) (Figure 1c), the influence on $D_{\mathrm{h}}$ of the diminished pulse repetition rate and dose per pulse is not very significant for the base PVP systems or for $\mathrm{P}^{*}$-g-A (200) because of the high dilution of potentially reactive species. It becomes quite important at the higher 
Table 1. Attribution of Main ${ }^{13} \mathrm{C}$ NMR Chemical Shifts of Nonirradiated (Linear) PVP, APMAM Monomer, and P*-gA(50) L80 MG

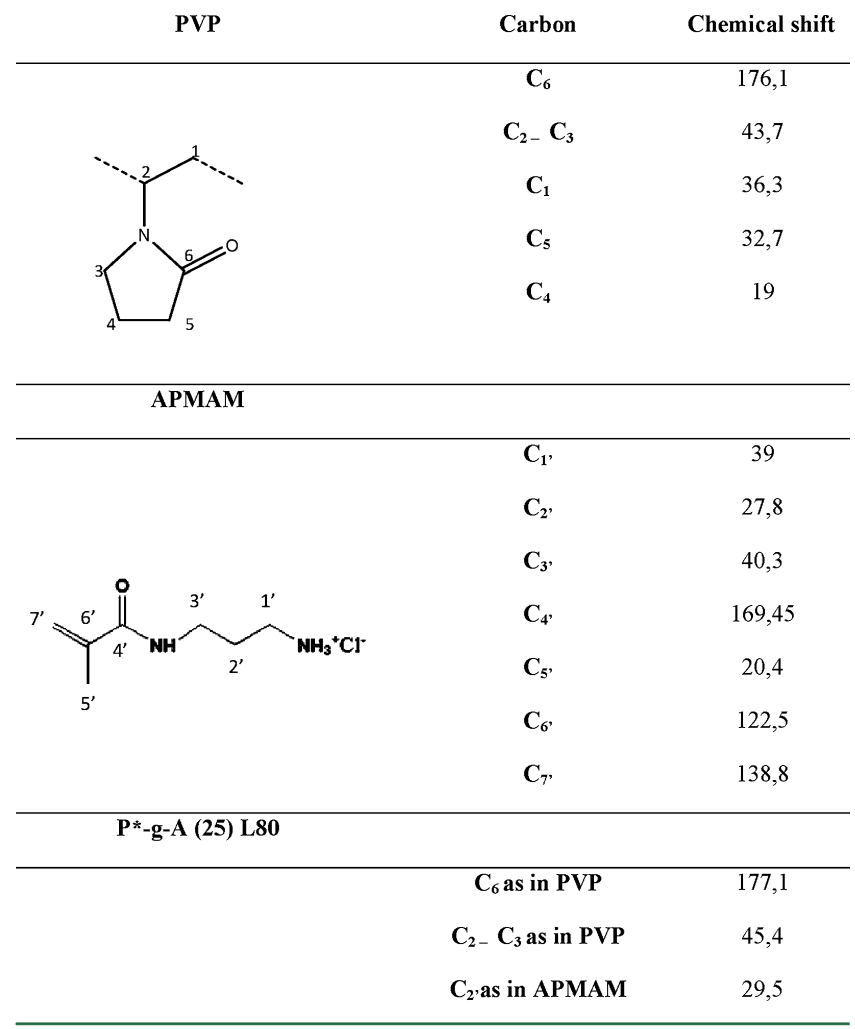

Table 2. XPS Surface Quantitative Analysis of the Investigated Samples ${ }^{a}$

\begin{tabular}{lccccc}
\multicolumn{1}{c}{ system } & C 1s & O 1s & N 1s & O/C & N/C \\
PVP & 76.1 & 12.9 & 11.0 & 0.169 & 0.144 \\
P* L80 & 70.4 & 23.0 & 6.6 & 0.327 & 0.094 \\
P*-g-A (50) L80 & 68.7 & 22.9 & 8.4 & 0.333 & 0.122 \\
P* E80 & 70.7 & 22.0 & 7.3 & 0.311 & 0.103 \\
P*-g-A (50) E80 & 72.9 & 20.1 & 7.0 & 0.276 & 0.096 \\
P*-g-A (100) E80 & 61.2 & 31.4 & 7.4 & 0.513 & 0.121
\end{tabular}

${ }^{a}$ Elemental concentration is expressed as atomic percentage (at \%).

Table 3. XPS Surface Distribution of Carbon Species Resulting from the Curve-Fitting of $\mathrm{C}$ 1s Photoelectron Peak $^{a}$

\begin{tabular}{cccc} 
& \multicolumn{3}{c}{ C 1sBE $(\mathrm{eV})$} \\
\cline { 2 - 4 } system & 285.1 & 286.5 & 288 \\
PVP & 69.8 & 20.5 & 9.7 \\
P* L80 & 67.8 & 12.9 & 19.4 \\
P*-g-A (50) L80 & 64.6 & 16.9 & 18.5 \\
P* E80 & 66.1 & 16.3 & 17.6 \\
P*-g-A (50) E80 & 72.4 & 10.6 & 17.0 \\
P*-g-A (100) E80 & 67.5 & 15.0 & 17.5 \\
${ }^{a}$ Carbon species are expressed as peak area percentage.
\end{tabular}

APMAM concentrations, where the switch from mainly intramolecular modification to interparticle bridging leading to microparticle formation occurs already for the 1:100 PVP RU/APMAM molar ratio. Reducing the dose rate (i.e., frequency) makes the time lag between two successive pulses
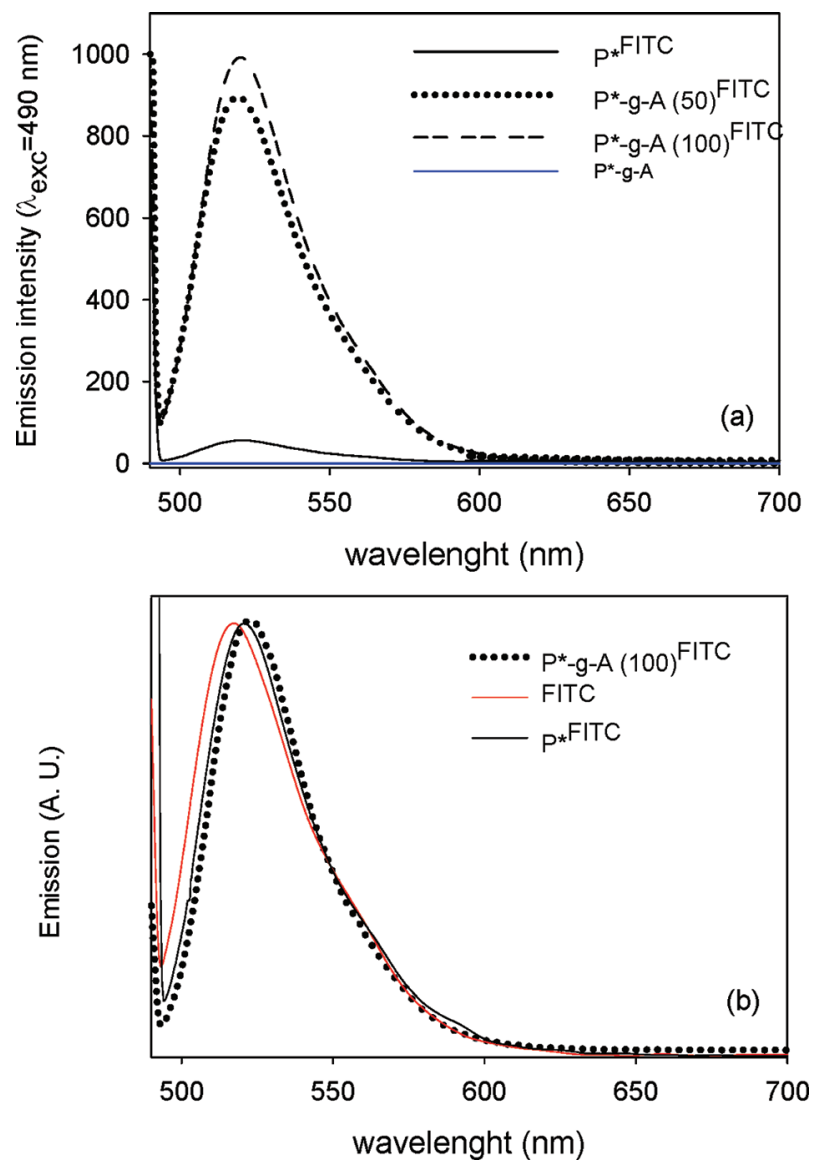

Figure 5. UV-vis emission spectroscopy of: (a) Nonconjugated amino-functionalized nanogels, two FITC-conjugated variants, and corresponding FITC-incubated base PVP nanogel (irradiation condition: E80). (b) Normalized emissions of a typical FITCconjugated amino-grafted nanogels/microgel $\left(\mathrm{P}^{*}\right.$-g- $\left.\mathrm{A}^{\text {FITC }}\right)$, FITCincubated base PVP nanogels $\left(\mathrm{P}^{* \mathrm{FITC}}\right)$, and free FITC aqueous solution (FITC).

Table 4. Emission Intensity at $\lambda_{\text {peak }}$ for the FITC Conjugated Systems

\begin{tabular}{|c|c|}
\hline system & emission intensity at peak (A.U.) \\
\hline $\mathrm{P} * \mathrm{E} 40$ & 55 \\
\hline $\mathrm{P}^{*}$-g-A (100) E40 & 450 \\
\hline $\mathrm{P}^{*}$-g-A(50) E40 & 202 \\
\hline $\mathrm{P} * \mathrm{E} 80$ & 50 \\
\hline $\mathrm{P}^{*}$-g-A (100) E80 & 990 \\
\hline $\mathrm{P}^{*}-\mathrm{g}-\mathrm{A}(50) \mathrm{E} 80$ & 890 \\
\hline $\mathrm{P} * \mathrm{~L} 80$ & 60 \\
\hline $\mathrm{P}^{*}$-g-A (100) L80 & 446 \\
\hline $\mathrm{P}^{*}$-g-A(50) L80 & 105 \\
\hline
\end{tabular}

comparable to the time scale of encounters of potentially reactive species, thus favoring particle size buildup.

Surface Charge Density of Base and Amino Grafted PVP Micro/Nanogels. Micro- and nanogels in suspension exhibited electrostatic charges on their surfaces that can be expressed as $\zeta$-potential and can be used to predict mutual interactions and thereby the long-term stability. In fact, particle flocculation and aggregation is less likely to occur in suspensions with higher $\zeta$-potential. Furthermore, a role is played by nanoparticles' surface charge in their entry through the cells membrane, their intracellular pathway, and fate. ${ }^{28}$ In 

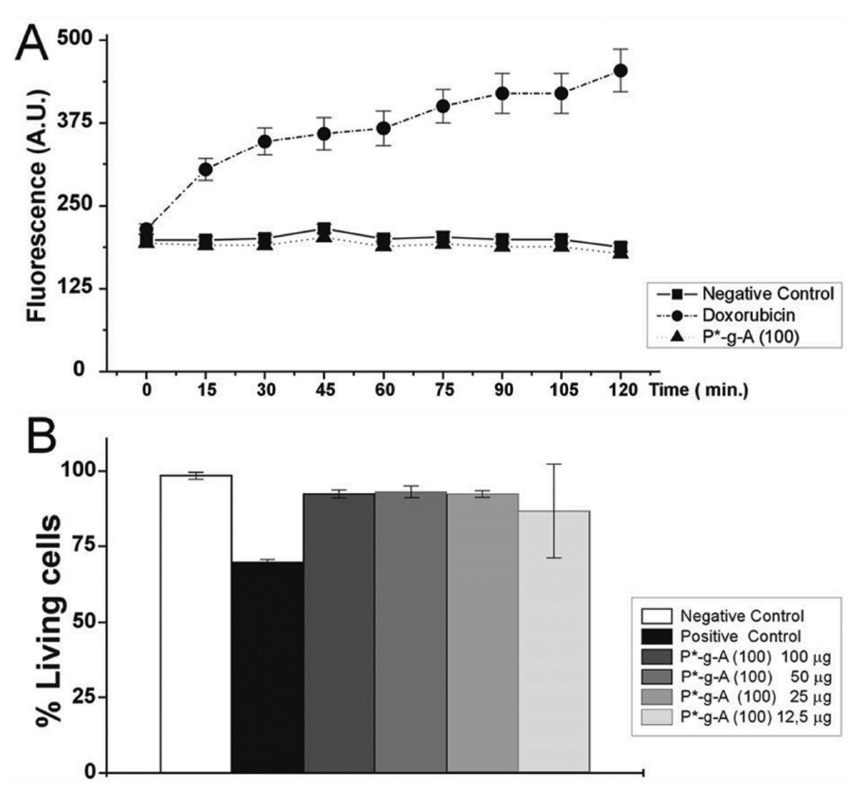

Figure 6. Cell viability by Caspase 3-7-8 enzymatic activation and MTT evaluation. (A) Activated caspases profiles obtained by spectrofluorimetric evaluation of Ac-Asp-Glu-Val-Asp-MCA peptide digestion at different times (from 0 to $120 \mathrm{~min}$ ). In the Figure (-口-) represents untreated MC 3T3-E (negative control); (-๑-) represents the MC 3T3-E1 DXR-treated cells (positive control); and ( $\cdots \mathbf{\Delta} \cdots)$ represents MC 3T3-E1 cells in the presence of $\mathrm{P}^{*}$-g-A (100) NG dispersion. In ordinate, the fluorochrome emission is reported as arbitrary units. (B) MC 3T3-E1 cell viability as metabolic respiration. In ordinate, the amount of living cells in $\%$ with respect to nontreated cells.

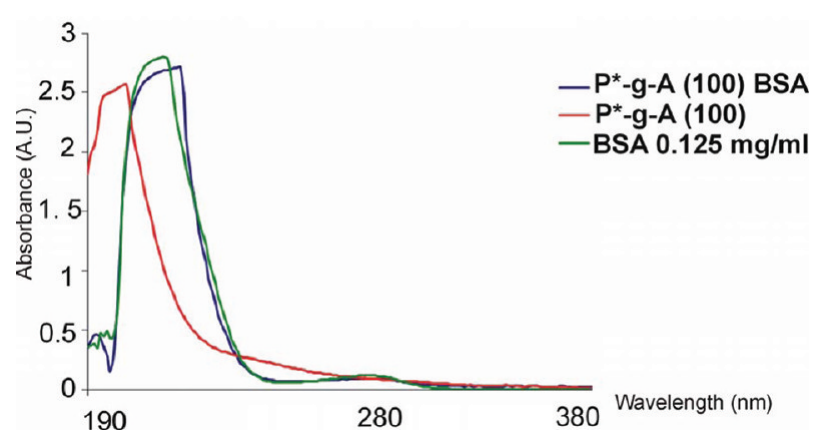

Figure 7. UV-vis absorption spectra of BSA-conjugated $\mathrm{P}^{*}$-g-A(100) system; nonconjugated $\mathrm{P}^{*}$-g-A(100) system as control; free BSA as reference.

Figure 2, $\zeta$-potentials of E40 and E80 systems are shown. It has been pointed out that the nonirradiated PVP at the same concentration ( $0.1 \mathrm{wt} \%)$ shows a slightly anionic character. Conversely, the irradiated base systems present a marked anionic behavior, more pronounced at the higher dose. (See Figures S4 and S5 for the entire $\zeta$-potential curves in the SI.) The average surface charge density is above $-30 \mathrm{mV}$, with a wide distribution of values that span from -15 to $-55 \mathrm{mV}$. (See Figure S6 in the SI.) It is worth pointing out that this distribution reflects the inherent heterogeneity of charge density for the MGs or NGs constituting the sample. The anionic character is likely to be induced by a change in the chemical structure and functionality of PVP upon irradiation under these conditions. This important aspect will be the subject of a dedicated paper, and some spectroscopic evidence of the irradiated base "L80" system will be anticipated in the next paragraph. NGs obtained from PVP in the presence of increasing amounts of APMAM show a progressively reduced anionic character, likely due to the already proposed screening effect of the negative charges of PVP by the protonated amino groups of APMAM. At the highest concentration of APMAM, when MGs are formed from aggregation of several PVP chains, this monomer or its oligomers are expectedly no longer preferentially located at the particles surface but in their interiors; therefore, $\zeta$-potential values become comparable to that of the base PVP systems.

Structural Characterization of Amino-Functionalized PVP Micro/Nanogels. Spectroscopic confirmation of the chemical attachment of APMAM to PVP has been sought by a combination of different spectroscopic techniques. In particular, in Figure 3, FTIR spectra of nonirradiated PVP, P*, P*-g$\mathrm{A}(50)$, and $\mathrm{P}^{*}-\mathrm{g}-\mathrm{A}(25)$ are presented. The spectrum of AMPAM is also reported for comparison. All of the irradiated systems present: (i) higher absorptions in the high wavenumber range of the spectrum $\left(3700-3000 \mathrm{~cm}^{-1}\right)$, where stretching vibration modes of hydroxyls $(\nu \mathrm{O}-\mathrm{H})$ and amidic $(\nu \mathrm{N}-\mathrm{H})$ groups and their associations are evident and (ii) an important modification of the amide I band $(\nu \mathrm{C}=\mathrm{O})$, generally at 1661 $\mathrm{cm}^{-1}$ and related to carbonyl stretching of pyrrolidone groups, with two new bands appearing at 1695 and $1765 \mathrm{~cm}^{-1}$. These bands possibly refer to the formation of an imide and precisely refer to the symmetric and asymmetric stretching of fivemembered cyclic imide. ${ }^{29,30}$ Furthermore, the enlargement of the amide I band toward lower wavenumbers and a new band at $1388 \mathrm{~cm}^{-1}$ strongly suggests the formation of carboxylate anions (asymmetric and symmetric carboxylate anion stretch at 1600 and $1385 \mathrm{~cm}^{-1}$, respectively), ${ }^{31}$ which may explain both the low $\mathrm{pH}$ measured and the anionic surface charges for these nanoparticles' dispersions. (See also Figure S8 of the SI.) A possible reaction pathway for the proposed structural modifications of irradiated PVP is provided as Figure S9 in the SI. When irradiation is carried out in the presence of APMAM, differences with respect to the base $\mathrm{P}^{*}$ system are subtle. A relative increase of the absorption related to the amide I band of PVP and a shoulder at $1537 \mathrm{~cm}^{-1}$ can be noticed, probably due to the contribution of amide/amine bands of APMAM ${ }^{30}$ The strong peak at $1610 \mathrm{~cm}^{-1}$ present in the free monomer and mainly related to double-bond stretching vibration is not present in any of the irradiated systems or any of the corresponding characteristic bands at 1013, 940, and $811 \mathrm{~cm}^{-1}$. This last evidence suggests a radical addition reaction of APMAM to PVP.

Solid-state ${ }^{13} \mathrm{C}$ NMR has been carried out on the $1.5 \mathrm{P}^{*}$-g$\mathrm{A}(25) \mathrm{L} 80$ and on the linear PVP and monomeric APMAM, as reference. (See Figure 4 and Table 1.) Chemical shifts with reference to those of both pure PVP and APMAM were assigned according to literature data ${ }^{29,32}$ and are reported in Table 1, where major peaks for MG are also reported. Modification of the spectral features of the MG with respect to the linear PVP is evident in all of the different carbon sites. Although the obtained spectrum is not suitable for an exact identification and quantification of the different species, the evident shift of the carbonyl band toward the higher chemical shifts is in good agreement with the proposed structural modifications of PVP.

The influence of the electron beam irradiation on the surface chemical composition of the produced MGs and NGS was also investigated by X-ray photoelectron spectroscopy (XPS) due to 

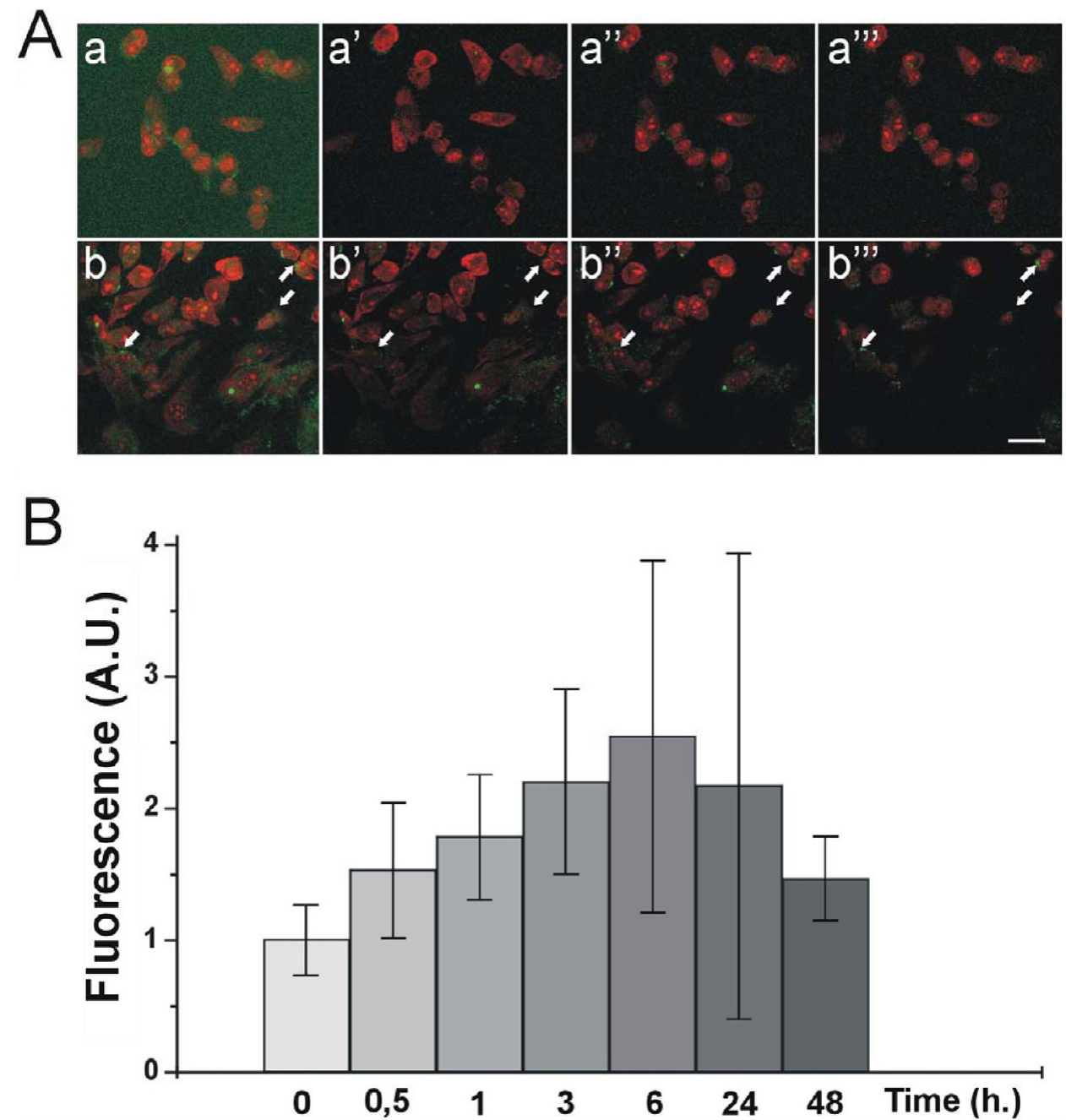

Figure 8. Localization of $\mathrm{P}^{*}$-g-A $(100)^{\mathrm{FITC}} \mathrm{NGs}$ in MC3T3-E1 cells. (A) Confocal analysis of MC3T3-E1 cells stained with EtBr (red) in the presence of $\mathrm{P}^{*}-\mathrm{g}-\mathrm{A}(100)^{\mathrm{FITC}} \mathrm{NGs}$ (green) at $30 \mathrm{~min}\left(\mathrm{a}-\mathrm{a}^{\prime \prime \prime}\right)$ and $6 \mathrm{~h}\left(\mathrm{~b}-\mathrm{b}^{\prime \prime \prime}\right)$. Panels $(\mathrm{a}, \mathrm{b})$ show the images obtained by resumption of all sections, with an overall thickness of $\sim 15 \mu \mathrm{m}$; in the panels $\left(\mathrm{a}^{\prime}-\mathrm{b}^{\prime}\right),\left(\mathrm{a}^{\prime \prime}-\mathrm{b}^{\prime \prime}\right)$, and $\left(\mathrm{a}^{\prime \prime \prime}-\mathrm{b}^{\prime \prime \prime}\right)$ sections were at a depth of 3,6 , and $10 \mu \mathrm{m}$, respectively. Arrows in $\left(\mathrm{b}-\mathrm{b}^{\prime \prime \prime}\right)$ indicate the positions of $\mathrm{P}^{*}$-g-A $(100)^{\mathrm{FITC}}$ NGs inside the cells. Scale bar is $20 \mu \mathrm{m}$. (B) Histogram represents the amount of fluorescent nanoparticles present inside the MC3T3-E1 cells at different times (from 0 to $48 \mathrm{~h}$ ). In ordinate, the fluorescence emission is reported as arbitrary units.

its ability to provide surface quantitative elemental and chemical state information. The surface chemical composition of the investigated samples is reported in Table 2, and quantitative results are expressed as atomic percentage (at \%). A sample of nonirradiated PVP was taken as reference. Differences in atomic percentage are detected before and after irradiation of aqueous PVP. A noticeable increase in the oxygen concentration and a slight decrease in the carbon and nitrogen amounts are evidenced on the surface of the irradiated PVP samples $\left(\mathrm{P}^{*}\right)$. The value of the $\mathrm{O} / \mathrm{C}$ ratio increases from 0.169 to 0.327 after irradiation with LAE at $80 \mathrm{kGy}$, and the N/ $\mathrm{C}$ decreases from 0.144 to 0.094 , as reported in Table 2. These results confirm an increased level of PVP oxidation upon irradiation, which is consistent with the findings of the other spectroscopic techniques applied. No significant differences were observed in surface quantitative analysis among the samples subjected to irradiation at different dose rates or in the presence of APMAM, with the only exception of P*-g-A (100) E80. This sample shows the highest $\mathrm{O} / \mathrm{C}$ ratio and a slightly higher N/C with respect to P*-g-A (50) E80 despite the fact that it comes from a halved amount of APMAM in the feed.
A nonlinear least-squares curve fitting routine was used for the analysis of XPS spectra, separating elemental species in different oxidation states. In all of the investigated samples the $\mathrm{N}$ 1s photoelectron peak consists of a single component located at $\mathrm{BE}=400.1 \mathrm{eV}$ that can be attributed to nitrogen species in the amide group $\mathrm{N}-\mathrm{C}=\mathrm{O} .{ }^{33}$ In all $\mathrm{O}$ 1s spectra, the component of the photoelectron peak corresponding to the $\mathrm{BE}$ of the carbonyl group is located at $\mathrm{BE}=531.5 \mathrm{eV}$, which is assigned to oxygen species in $\mathrm{N}-\mathrm{C}=\underline{\mathrm{O}} .{ }^{33,34}$ The curve-fitting of the $\mathrm{C} 1 \mathrm{~s}$ photoelectron peak revealed the presence of three components at $\mathrm{BE}=285.1,286.5$, and $288.1 \mathrm{eV}$, which can be assigned to carbon atoms in $\mathrm{C}-\mathrm{C}, \mathrm{C}-\mathrm{O}$, and $\mathrm{C}=\mathrm{O}$ bonds, respectively. ${ }^{34,35}$ Results of the curve-fitting procedure of $\mathrm{C} 1 \mathrm{~s}$ peak are reported in Table 3 . The relative amount of $\mathrm{C}-\mathrm{O}$ and $\mathrm{C}=\mathrm{O}$ species in nonirradiated PVP well describes the tautomerism of the pyrrolidone group in the linear polymer. A different content of $\mathrm{N}-\underline{\mathrm{C}}=\mathrm{O}$ carbon species, corresponding to $\mathrm{C} 1 \mathrm{~s}$ component at $\mathrm{BE}=288.1 \mathrm{eV}$, is detected in the base PVP samples before and after irradiation. Electron beam irradiation induces a significant increase in the concentration of carbon species in the amide group, coherently with the 
proposed formation of a cyclic imide. The increase of these species are confirmed by the already discussed increase of the $\mathrm{N}-\mathrm{C}=\mathrm{O}$ species in the $\mathrm{O} 1 \mathrm{~s}$ spectra.

Conjugation of FITC to Amino-Functionalized PVP Micro/Nanogels. Chemical conjugation of FITC to the amino-functionalized MGs and NGs has been attempted to determine whether the primary amines are available for chemoligation and also to generate fluorescent variants for biological evaluation in vitro. UV-visible absorption and emission spectroscopies have been used to assess the success of conjugation and to quantify the amount of bound FITC. In particular, in Figure 5a the emission spectra of fluorescent E80 MGs and NGs are reported (1:50 and 1:100, respectively). A nonconjugated system and the FITC-incubated base $\mathrm{P}^{*}$ are also shown. Both the base $\left(\mathrm{P}^{*}\right)$ and amino-graft nonconjugated systems ( $\mathrm{P}^{*}$-g-A) do not present any significant emission band when excited at $490 \mathrm{~nm}$, whereas P*FTIC systems show a modest emission. Figure $5 \mathrm{~b}$ reports the normalized emissions of FITC-conjugated $\mathrm{P}^{*}$-g-A, FITC-incubate $\mathrm{P}^{*}$, and free probe. Normalization allows easier identification of shifts in the $\lambda_{\text {peak }}$. It is evident that the emission band of a FITC-conjugated aminografted MG or NG is similar to that of the free probe with a significant red shift $(+5 \mathrm{~nm})$, which confirms the formation of thioureic bond. ${ }^{36}$ The P*FTIC systems show a modest emission band, with a peak at an intermediate position between that of the free and the bound probe. This emission is likely caused by FITC physically entrapped in the cross-linked particles. A previous work of some of the authors has established that PVP macrogels produced through e-beam irradiation have hydrophobic pockets that favorably, and in some circumstances also irreversibly, house fewer polar molecules present in aqueous systems. $^{4}$

Absorption spectra for conjugated systems and free FITC solutions are reported as Figures S10 and S11 of the SI. Like in the emission spectra, the maximum absorption peak of the conjugated micro/nanogels is shifted with respect to the free FITC solution. Furthermore, $\zeta$-potential measurements carried out on all conjugated systems show narrower charge distributions, centered around neutrality, thus suggesting a modification of the surface charge distribution. (See Figure S7 in the SI.) All this evidence confirms the success of conjugation.

Emission intensities at the peak for $1: 100$ and 1:50 $\mathrm{P}^{*}$-g-A systems and the corresponding base $\mathrm{P} * \mathrm{FTIC}$ NGs under the different irradiation conditions are reported in Table 4 . The emission intensity of the $\mathrm{P}^{*}-\mathrm{g}-\mathrm{A}^{\mathrm{FTIC}}(100)$ systems is higher than that of $\mathrm{P}^{*}$-g-A $\mathrm{A}^{\mathrm{FTIC}}(50)$, despite the two-fold increase in APMAM moles in the formulation for the latter. $\mathrm{P}^{*}$-g-A(100) systems are genuinely NGs, whereas $\mathrm{P}^{*}-\mathrm{g}-\mathrm{A}(50)$ systems are MGs under all investigated irradiation conditions. If the formation of bigger particles is involving APMAM as a coupling agent, then a relevant amount of the amino groups may not be accessible to FITC or may have been depleted by the establishment of strong interactions with PVP.

FITC detachment was performed for $\mathrm{P}^{*}-\mathrm{g}-\mathrm{A}(100)^{\mathrm{FTIC}}$ and $\mathrm{P}^{*}$-g-A $(50)^{\mathrm{FTIC}} \mathrm{E} 80$ to attempt a quantitative estimate of the content of primary amino groups available for chemoligation. A molar extinction coefficient for "bound FITC" was experimentally determined (see the SI for details), and the moles of available amino groups $\left(4.4 \times 10^{-4}\right.$ and $\left.1.3 \times 10^{-4} \mathrm{mM}\right)$ represent about 0.5 and $0.3 \%$ of the moles of APMAM charged in the feed for $\mathrm{P}^{*}-\mathrm{g}-\mathrm{A}(100)$ and $\mathrm{P}^{*}$-g-A(50), respectively. Despite the fact that only a low proportion of the theoretical amino groups present are available, the FITC-conjugated variants enabled NG visualization in vitro cells studies.

Biological Evaluation. PVP is a FDA approved, biocompatible polymer widely used in pharmaceutical applications. In particular, cross-linked PVP nanoparticles have already been evaluated for drug and gene delivery. ${ }^{37,38}$ It has also been shown that these particles can be administered intravenously and can remain in circulation for a considerable period of time evading the immune system. ${ }^{39}$ Biological assays were here performed on cells treated with NGs to verify their biocompatibility. In particular, we report in detail the results obtained with $\mathrm{P}^{*}$-g-A(100) E80. To exclude apoptotic events, we performed an enzymatic assay on caspases activation. (See Materials and Methods sections.) As shown in Figure 6A, MC3T3-E1 cells treated or not (negative control) with the NGs at different times were compared with the ones treated with DXR (positive control). NG-treated cells did not show any activation of enzymes, whereas time-dependent fluorochrome release was observed in DXR-treated cells. A morphological evaluation, by confocal microscopy, was also carried out on MC3T3-E1 cells incubated in the presence of NGs for $24 \mathrm{~h}$ and stained with acridine orange/EtBr solution (data not shown); the staining allows us to discriminate among alive, damaged, and dead cells. This observation is in agreement with the enzymatic assay; in fact, cells treated with NGs do not show any apoptotic signs, equally to untreated cells used as negative control. Differently, the typical signs of apoptosis were observed in the positive control in which cells were treated with $\mathrm{DXR}$, known to be an apoptosis inducer.

Once the absence of cytotoxicity of NGs was established, a cell viability assay was performed to assess if these nanoparticles influence cellular metabolism. As it is possible to observe in Figure 6B, MC3T3-E1 cells treated with different concentrations of NG aqueous dispersion have a similar profile to untreated cells; differently, DXR-treated cells (positive control) show a mortality of $\sim 30 \%$.

As all gathered data suggest a good degree of biocompatibility of the produced NGs, experiments of biological molecules conjugation were performed to assess NGs' potential as smart drug delivery systems. In particular, aiming to bind stably biological molecules, such as proteins or antibodies, for active targeting, we performed conjugation experiments using BSA as a model. Absorption spectra reported in Figure 7 show that the BSA-conjugated NG spectrum has a peak at $280 \mathrm{~nm}$ that coincides with the one of free BSA, used as reference, whereas no absorption peak was observed in the nonconjugated system. The interaction between BSA and nanoparticles is fairly strong as it survives upon extensive dialysis and repeated washings. It was estimated that the weight percentage of retained BSA per polymer is $\sim 30$, and, in consideration of the dimensions of BSA, we expect the protein to be located at the nanoparticles' surface.

To test nanoparticles viability thought cell compartments, we used the fluorescent $\mathrm{P}^{*}$-g-A(100) $)^{\mathrm{FITC}}$ variant. MC3T3-E1 cells were treated with the NGs for different incubation periods to follow their localization inside and through the cells. Figure 8A $\left(a-a^{\prime \prime \prime}\right)$ shows that after $30 \mathrm{~min}$ of incubation NGs were present in the media and on the cells' surface, whereas after $6 \mathrm{~h}$ of incubation (Figure 8A $\left.\left(b-b^{\prime \prime \prime}\right)\right)$, NG distribution in cytoplasm compartment was mostly evident; similarly, in samples incubated for 24 and $48 \mathrm{~h}$, cytoplasmic distribution was still evident (data not shown), although in lower amount. NGs' secretion as waste products or their digestion by the 
lysosomal cell system could be hypothesized. In vivo and ex vivo tests will be performed to elucidate this aspect. Spectrofluorimetric measurements on the incubated cells after extraction and purification from the culture medium confirm the results of confocal microscopy. Figure $8 \mathrm{~B}$ relates the amount of $\mathrm{P}^{*}$-g-A $\mathrm{A}^{\mathrm{FITC}} \mathrm{NGs}$ inside or attached to MC3T3-E1 cells to the incubation time. A maximum of fluorescence has been found after $6 \mathrm{~h}$ of incubation, corresponding to $6 \%$ of the loaded amount, which decreases in $24 \mathrm{~h}$ and further in $48 \mathrm{~h}$. Measurements of fluorescence from the culture medium and washings nicely balance the changes in emissivity from the cells, as shown in Figure S12 of the SI.

\section{CONCLUSIONS}

We believe that the work presented here can strongly encourage us to pursue using e-beam radiation processing as a viable and effective synthetic methodology to manufacture functional nanocarriers in a single step and with high yields. After irradiation, no expensive or time-consuming purification procedures are required, as no recourse to catalysts, initiators, or surfactants is made. Simultaneous sterilization can also be achieved by a proper selection of the irradiation doses. In particular, cross-linked PVP grafted with APMAM, a monomer bearing primary amino groups, has been chosen to demonstrate the potential of the approach. The analyzed $\mathrm{P}^{*}$-g-A(100) NGs are biocompatible and able to bind biological molecules stably. Cellular internalization experiments showed a significantly enhanced cellular accumulation of NGs, probably by an endocytic pathway: a direct translocation process without observable cytotoxic processes. This study offers a very exciting prospect in the use of the generated nanoparticles as potential drug delivery carriers.

\section{ASSOCIATED CONTENT}

\section{S Supporting Information}

The following figures are provided: DLS autocorrelation function curves for a representative NGs dispersion after dialysis with different MWCO membranes (Figure S1); ${ }^{1} \mathrm{H}$ NMR spectra of nonirradiated and irradiated aqueous APMAM with LAE at $80 \mathrm{kGy}$ (Figure S2); DLS autocorrelation function curves for nonirradiated PVP (P), irradiated PVP E40 (P*), and irradiated PVP E40 (P*) after $0.22 \mu \mathrm{m}$ filtration (Figure $\mathrm{S} 3$ ); $\zeta$-potential curves for nonirradiated PVP in water (Figure S4) and for base PVP NGs (P* E80) in water (Figure S5), a typical amino-functionalized NG/MG and a FITC conjugated amino-functionalized system, $\left(\mathrm{P}^{*}\right.$-g-A $(50)^{\mathrm{FITC}}$ ) (Figures S6 and S7); Overlaid FTIR spectra of nonirradiated and irradiated PVP (Figure S8); reaction pathway to for the proposed structural modifications of irradiated PVP (Figure S9); normalized absorption spectra for a typical conjugated NG system and a free FITC solution (Figure S10); and experimental details and calculations for the quantitative determination of available amino groups through FTIC detachment experiments. Absorption spectra of a typical FTIC-conjugated NG (P*-g$\left.\mathrm{A}(100)^{\mathrm{FTIC}}\right)$, FITC solution collected under the filter after detachment, and conjugated NG recovered from the top of the filter (redispersed in PBS) after detachment (Figure S11); histogram representing the total fluorescence in the wells containing MC3T3-E1cells incubated with fluorescent NGs in their culture medium, and fluorescence signals from extracted cells, culture medium, and washings collected in cells extraction process, at different times (from 0 to $48 \mathrm{~h}$ ), as obtained from independent measurements. In ordinate, the fluorescence emission is reported as arbitrary units. Emission derives from the fluorescent NGs as they partition in the different locations as function of the time (Figure S12). This material is available free of charge via the Internet at http://pubs.acs.org.

\section{AUTHOR INFORMATION}

\section{Corresponding Author}

*Tel: +39 091 23863710. Fax: +39 091 23863738. E-mail: Clelia.dispenza@unipa.it.

\section{Notes}

The authors declare no competing financial interest.

\section{ACKNOWLEDGMENTS}

This work was partially supported by the IAEA CRP: "Nanoscale radiation engineering of advanced materials for potential biomedical applications" and FW7-COST Action MP0701.

We acknowledge the assistance of the Centre for Radiation Research and Technology, Institute of Nuclear Chemistry and Technology, (Warsaw) for e-beam irradiation. In particular, we gratefully thank Prof. A. Chmielewski, Prof. G. Przybytniak, and Prof. I. Kaluska for their support and advice.

Solid-state ${ }^{13} \mathrm{C}$ NMR experimental data were provided by Centro Grandi Apparecchiature UniNetLab-Università di Palermo, funded by P.O.R.Sicilia2000-2006, Misura 3.15 Quota Regionale.

\section{REFERENCES}

(1) Hoffman, A. S. Adv. Drug Delivery Rev. 2002, 43, 3-12.

(2) Kopecek, J. Biomaterials 2007, 28, 5185-5192.

(3) Peppas, N. A.; Bures, P.; Leobandung, W.; Ichikawa, H. Eur. J. Pharm. Biopharm. 2000, 50, 27-46.

(4) Ricca, M.; Foderà, V.; Giacomazza, D.; Leone, M.; Spadaro, G.; Dispenza, C. Colloid Polym. Sci. 2010, 288, 969-980.

(5) Oishi, M.; Nagasaki, Y. Nanomedicine 2010, 5, 451-468.

(6) Vinogradov, S. V. Nanomedicine 2010, 5, 165-168.

(7) Liu, Y.; Miyoshi, H.; Nakamura, M. Int. J. Cancer. 2007, 120, 2527-2537.

(8) Moghimi, S. M.; Hunter, A.-C.; Murray, J.-C. Pharmacol. Rev. 2001, 53, 283-318.

(9) Ganta, S.; Devalapally, H.; Shahiwala, A.; Amiji, M. J. Controlled Release 2008, 126, 187-204.

(10) Jung Kwon, O.; Drumright, R.; Siegwart, D. J.; Matyjaszewski, K. Prog. Polym. Sci. 2008, 33, 448-477.

(11) Dispenza, C.; Grimaldi, N.; Sabatino, M.-A.; Todaro, S.; Bulone, D.; Giacomazza, D.; Przybytniak, G.; Alessi, S.; Spadaro, G. Radiat. Phys. Chem. 2011, DOI: 10.1016/j.radphyschem.2011.11.057.

(12) Ulanski, P; Janik, I.; Rosiak, J. M. Radiat. Phys. Chem. 1998, 52, 289-294.

(13) Kadlubowski, S.; Grobelny, J.; Olejniczak, W.; Cichomski, M.; Ulanski, P. Macromolecules 2003, 36, 2484-2492.

(14) Jeszka, J. K.; Kadlubowski, S.; Ulanski, P. Macromolecules 2006, 39, $857-870$.

(15) Stepanek, P. Data Analysis in Dynamic Light Scattering. In Dynamic Light Scattering: The Method and Some Applications; Brown, W., Ed.; Oxford University Press: Oxford, U.K., 1993; pp 177-240.

(16) Woods, R. J.; Pikaev, A. K. Applied Radiation Chemistry: Radiation Processing; John Wiley and Sons, Inc.: New York, 1994; Chapter 6.

(17) Musyanovych, A.; Adler, H.-J. P. Langmuir 2005, 21, 22092217.

(18) Sherwood, P. M. A. In Practical Surface Analysis; Briggs, D., Seah, M. P., Eds; Wiley: New York, 1990; p 181.

(19) Wagner, C. D.; Davis, L. E.; Riggs, W. M. Surf. Interface Anal. 1986, 2,5 .

(20) Frisken, B. J. Appl. Opt. 2001, 40, 4087-4091. 
(21) Carlfors, J.; Rymden, R. Eur. Polym. J. 1982, 18, 933-937.

(22) Nicholson, D. W.; Ali, A.; Thornberry, N. A.; Vaillancourt, J. P.; Ding, C. K.; Gallant, M.; Gareau, Y.; Griffin, P. R.; Labelle, M.; Lazebnik, Y. A. Nature 1995, 6, 37-43.

(23) Thornberry, N. A.; Rano, T. A.; Peterson, E. P.; Rasper, D. M.; Timkey, T.; Garcia-Calvo, M.; Nordstrom, P. A.; Roy, S.; Vaillancourt, J. P.; Chapman, K. T.; Nicholson, D. W. J. Biol. Chem. 1997, 18, 17907-11.

(24) An, J.-C.; Weaver, A.; Kim, B.; Barkatt, A.; Poster, D.; Vreeland, W. N.; Silverman, J.; Al-Sheikhly, M. Polymer 2011, 52, 5746-5755.

(25) Chapiró, A. Radiation Chemistry of Polymeric Systems; WileyInterscience: New York, 1962.

(26) Al-Assaf, S.; Phillips, G. O.; Deeble, D. J.; Parsons, B.; Starnes, H.; von Sonntag, C. Radiat. Phys. Chem. 1995, 46, 207-217.

(27) Ulanski, P.; Kadlubowski, S.; Rosiak, J. M. Radiat. Phys. Chem. 2002, 63, 533-537.

(28) Verma, A.; Stellacci, F. Small 2010, 6, 12-21 and references herein..

(29) Zhu, X.; Lu, P.; Chen, W.; Dong, J. Polymer 2010, 51, 30543063.

(30) Nakanishi, K. Infrared Absorption Spectroscopy; Holden-Dy: San Francisco, 1962.

(31) Silverstein, R. M.; Bassler, G. C.; Morrill, T. C. Spectrometric Identification of Organic Compounds, 3rd ed.; John Wiley \& Sons, Inc.: New York, 1974.

(32) Pretsch, E.; Buhlmann, P.; Badertscher, M. ${ }^{13} \mathrm{C}-\mathrm{Nuclear}$ Magnetic Resonance Spectroscopies. In Spectral Data for Structure Determination of Organic Compounds. Tables of Spectral Data; Springer: New York, 2009; pp 69-156.

(33) Moulder, J. F.; Stickle, W. F.; Sobol, P. E.; Bomben, K. D. Handbook of X-Ray Photoelectron Spectroscopy: A Reference Book of Standard Spectra for Identification and Interpretation of XPS Data; Chastain, J., King, R. C., Jr., Eds.; Physical Electronics, Inc.: Eden Prairie, MN, 1995.

(34) Sionkowska, A.; Wisniewski, M.; Kaczmarek, H.; Skopinska, J.; Chevallier, P.; Mantovani, D.; Lazare, S.; Tokarev, V. Appl. Surf. Sci. 2006, 253, 1970-1977.

(35) Thissen, H.; Johnson, G.; Hartley, P. G.; Kingshott, P.; Griesser, H. J. Biomaterials 2006, 27, 35-43.

(36) Lanz, E.; Gregor, M.; Slavik, J.; Kotyk, A. J. Fluoresc. 1997, 4, 317-319.

(37) Hamidi, M.; Azadi, A.; Rafiei, P Adv. Drug Delivery Rev. 2008, $60,1638-1649$.

(38) Saxena, A.; Mozumdar, S.; Johri, A. K. Biomaterials 2006, 27, 5596-5602.

(39) Bharali, D. J.; Sahoo, S. K.; Mozumdar, S.; Maitra, A. J. Colloid Interface Sci. 2003, 258, 415-23. 